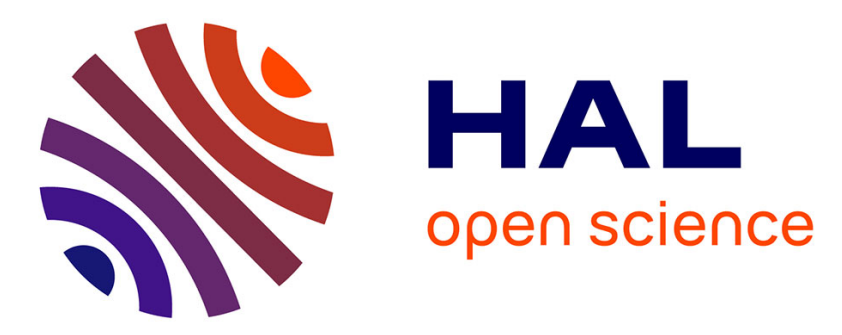

\title{
Historique et avenir des reboisements dans le département de la Loire \\ Marcel Nier
}

\section{To cite this version:}

Marcel Nier. Historique et avenir des reboisements dans le département de la Loire. Revue forestière française, 1952, 11, pp.714-727. 10.4267/2042/27914 . hal-03380188

\section{HAL Id: hal-03380188 \\ https://hal.science/hal-03380188}

Submitted on 15 Oct 2021

HAL is a multi-disciplinary open access archive for the deposit and dissemination of scientific research documents, whether they are published or not. The documents may come from teaching and research institutions in France or abroad, or from public or private research centers.
L'archive ouverte pluridisciplinaire HAL, est destinée au dépôt et à la diffusion de documents scientifiques de niveau recherche, publiés ou non, émanant des établissements d'enseignement et de recherche français ou étrangers, des laboratoires publics ou privés. 


\section{HISTORIQUE \\ ET AVENIR DES REBOISEMENTS DANS LE DÉPARTEMENT DE LA LOIRE}

Indice bibliographique: F 96 (44.58)

Si le Département de la Loire ne peut, malgré son réel intérêt forestier, peut-être trop méconnu, rivaliser avec les départements français les plus boisés, il est certainement l'un de ceux où l'effort de reboisement est le plus ancien et s'est exercé avec le plus de continuité et de ténacité.

\section{I. - Historique des Reboisements}

Vingt ans avant la première loi sur la restauration des montagnes du 28 juillet I860, le Garde Général des Eaux et Forêts de Saint-Etienne disait au Conseil Municipal de la Commune de Pelussin (Loire) alors peu acquis à la cause forestière:

" Vous avez encore plus de Ioo hectares de vides, qu'il faut boiser de toutes pièces: je vous propose de les ensemencer en pins, en épicéas, en mélèzes. Donnez-nous les crédits nécessaires, ioo francs par an pour commencer, et nous nous mettons au travail. ”

Ce n'étaient point là des paroles lancées à la légère. Un premier essai de semis réalisé en I843, après écobuage sur un sol cultivé au préalable pendant deux ans en blé et avoine, réussit parfaitement et fut renouvelé avec. le même succès en I 844 sur I hectare, ainsi qu'en 1845 , année où furent commencées les plantations de sapins, d'épicéas et de mélèzes, qui se continuèrent en 1846 et en I 847 , donnant une réussite évaluée à $93 \%$.

En I860, I 5 hectares étaient reboisés et une pépinière de 4 ares fonctionnait depuis deux ans au canton du Priel.

\section{$\mathrm{I}^{\circ}$ Périmètres consécutifs à la loi de 1860}

Ces essais parvinrent-ils à la connaissance du Conseil Général? Toujours est-il que cette Assemblée renouvela en I858 un vœu précédemment émis en faveur des montagnes de la Loire et le reprit l'année suivante. Dès l'apparition de la loi du 28 juillet I 860 , elle demanda que les mesures législatives complémentaires fussent prises pour la protection du gazonnement et contre les abus de la 
dépaissance et en I 862 elle vota une subvention de 3.000 francs à titre de contribution à l'œuvre de reboisement entreprise. Par la suite ellé inscrivit chaque année un crédit à son budget pour le même motif.

Encouragé par l'intérêt que le Conseil Général portait au reboisement, le Service forestier local, dès la publication de la loi de I860; se mit à l'œuvre pour l'étude de périmètres de reboisements et créa en I863 une pépinière de 3, Io ha à Saint-Etienne même: De I862 à I868, I3 périmètres d'une surface totale de 4.I2I ha, dont $\mathrm{I} .828$ ha en terrains communaux et d'établissements publics et '2.293 ha en terrains particuliers, furent constitués.

La constitution de ces périmètres, pour laquelle l'Administration des Eaux et Forêts avait pouvoir complet d'appréciation, ne fut pas sans soulever des protestations, des terres de culture et des pâtures ayant été englobées dans les surfaces à reboiser. Les communes visées, dont la population était relativement importante par rapport à leur surface et à la valeur de leurs sols, craignirent surtout d'être contraintes à une diminution de leur cheptel vif; faute de pouvoir lui assurer une nourriture suffisante. Qu'il suffise de citer un extrait d'une délibération du Conseil Municipal de la Commune du Bessat, en date du I3 juillet I862:

«Considérant que les páturages de la commune du Bessat soumis au reboisement sont l'unique moyen d'existence des habitants de cette localité...

Qu'une fois que le reboisement de leurs terrains sera effectué, les pâturages seront supprimés,...

Que dès lors que les pâturages seront supprimés, les habitants ne pourront plus avoir de bestiaux,

Que, s'ils n'ont plus de bestiaux, ils n'auront plus d'engrais pour les terres et pour les prairies,

Et que, s'ils n'ont plus d'engrais, que deviendra la culture sur un sol ingrat qui ne produit de minimes récoltes qu'à force d'engrais?...

Ils s'opposent en conséquence au projet de reboisement sur le territoire de la commune du Bessat.

Et des extraits de rapports du Sous-Inspecteur des Eaux et Forêts de l'époque:

Rapport du 15 juin 1863: «Ayant constaté qu'il nous était impossible d'obtenir une réponse en nous transportant aux domiciles des habitants auxquels appartiennent les terrains à reboisęr sur la commune de Rochetaillée, ayant reconnu que tous refusaient de dire leur nom, j'ai décidé qu'hier is juin, nous nous tranporterions à la Commune où, par suite de la fête, nous serions sûr de trouver une grande partie des habitants. Une première visite a été pour le Maire qui était sorti. Après de longues recherches, le brigadier a fini par apprendre qu'il était malade et couché; mais, il est certain que ce magistrat était debout et dehors au moment de notre arrivée. Nous nous sommes néanmoins transporté sur la place de l'Eglise. Là tous sans exception $_{2}$ sans vouloir donner leur nom, ont déclaré qu'ils ne voulaient pas reboiser, 8

Rapport du 15 juillet 1882 concernant la commune du Bessat:

« Les premiers travaux de reboisement donnèrent lieu de la part, soit des habitants, soit de l'Autorité Múnicipale à une vive opposition qui fálllit se traduire par des voies de fait." 
La nécessité d'éviter la dégradation des sols, qui avait été un des principaux motifs de la loi du 28 juillet I860, n'apparaissait pas, il est vrai, dans le départemerf́t de la Loire d'une manière aussi évident que dans les Alpes, la phase active de l'érosion étant achevée dans les montagnes plus arrondies du Massif Central. Les rapports du Sous-Inspecteur de Saint-Etienne, qui, en exécution de la loi du 4 avril I 882 , procéda à la révision des périmètres de reboisements, mentionnent presque tous que " les terrains compris dans les périmètres, sont couvert de bruyères et de myrtilles entremêlées de diverses plantes herbacées qui, au point de vue soit du maintien des terres, soit du ralentissement dans l'écoulement des eaux fluviales ou provenant de la fonte des neiges, sont d'une efficacité bien supérieure à celle d'un simple gazonnement de graminées ». Cette simple phrase explique d'ailleurs pourquoi la loi du 8 juin I864, sur le gazonnement des montagnes, dont M. D'ALVERNY disait, dans un projet manuscrit de lettre à M. le Sénateur AudIFFRED, “ qu'elle n'était pas sérieuse, et qu'en réalité elle avait été faite pour masquer sous l'étiquette " gazonnement ) le premier recul du législateur devant les oppositions soulevées par la loi du reboisement de I860 ") n'a pas reçu d'application dans la Loire.

Des résultats n'en furent pas moins acquis et, en I882, 948 ha, dont 499 ha en terrains communaux et d'établissements publics et 449 ha en terrains particuliers, étaient reboisés.

C'est de cette période (I860-I882) que datent les reboisements qui ont conduit à la constitution des peuplements d'épicéas les plus âgés de la forêt de la Ville de Saint-Etienne, de la forêt des Hospices de Saint-Etienne et de la forêt communale d'Ambierle, ainsi que les peuplements de pins sylvestres des forêts sectionales de Saint-Bonnet-des-Quarts.

En mème temps, s'étaient poursuivis, en dehors des périmètres constitués, les reboisements communaux de Pélussin qui en I882, couvraient une surface de I 55 hectares dont 95 hectares à La Madeleine et 60 hectares à Neufond, et avaient été effectués, sur une cinquantaine d'hectares, des reboisements dans les forêts sectionales d'Ecoche, entre r86r et r865.

En définitive, c'est à 700 hectares environ qu'il faut évaluer la surface des terrains communaux et d'établissements publics reboisés de I 860 à I 882 .

Les propriétaires particuliers, de leur côté, ne restèrent pas inactifs, malgré l'hostilité qui se manifesta contre les périmètres de reboisements. Les uns exécutèrent eux-mêmes leurs travaux, les autres en laissèrent la charge à l'Etat. Mais en aucun cas, il ne fut recouru au procédé de l'expropriation prévu par la loi de I86o. Presque toujours des subventions de l'Etat (graines, plants et primes en argent) et du département (primes en argent) dont le mon- 
tant était variable et pouvait s'élever jusqu'à $75 \%$ du coût des travaux, furent accordées. La procédure des reboisements facultatifs fut en somme appliquée dans le cadre des périmètres des reboisements, à l'initiative de l'Administration des Eaux et Forêts.

Les plants qui furent attribués en subvention aussi bien aux collectivités qu'aux propriétaires venaient pour la plupart de la pépinière de Saint-Etienne, ainsi que des pépinières de Vizille et de la Chartreuse dans l'Isère, de Saint-Appollinaire dans le Rhône qui, comme la Loire, dépendait alors de la Conservation de Grenoble. Celle de Saint-Etienne avait, à la fin de I882, déjà produit plus de I 2 millions de plants de toutes essences: résineux surtout (pins sylvestres, pins laricios de Corse, pins noirs d'Autriche, épicéas, sapins, mélèzes, etc...) et feuillus (robiniers faux-acacias, chênes, châtaigniers, frênes, érables sycomores). Des plants, de pins sylvestres en général, furent aussi expédiés par les pépinières de l'Est de la France, notamment de la région de Nancy. Il est probable que ce sont ces derniers qui ont constitué certains peuplements de conformation souvent défectueuse dont les sujets diffèrent assez sensiblement de ceux de la race locale d'Auvergne.

\section{$2^{\circ}$ Période $1882-1950$}

Après la promulgation de la loi du 4 avril I882, une révision des périmètres établis en exécution de la loi du 28 juillet I86a fut effectuée. Aucun périmètre ne fut maintenu. Les terrains communaux et d'établissements publics reboisés furent seuls déclarés soumis au régime forestier. Les autres ainsi que tous les terrains particuliers reboisés ou non furent rendus à la libre disposition de leurs propriétaires.

Bien qu'il n'ait pas été constitué de nouveaux périmètres, les reboisements ne furent pas arrêtés. Ils semblent seulement s'être quelque peu ralentis de I882 à I900 aussi bien de la part des collectivités que des particuliers, si l'on remarque que pendant cette période, la pépinière de Saint-Etienne, qui fut la principale source d'approvisionnement en plants, n'en' livra guère plus de 5 millions.

C'est sans doute en raison de ce ralentissement qui a suivi la loi de I882 qu'en I89 I le conseil général de la Loire, toujours préoccupé de la question du reboisement, émit un vœu en vue de reboisements obligatoires dans les communes de Lérigneux, Roche, Saint-Bonnet-le-Courreau, Sauvain, Chalmazel (Monts du Forez) et le renouvela en I893. N'ayant pas obtenu satisfaction de l'Administration forestière, il chercha même en I893 à faire appel à l'initiative privée et nomma une commission chargée de provoquer la formation d'un syndicat de reboisement dont le but serait l'achat et le reboisement de terrains incultes à l'aide d'une alloca- 
tion annuelle du département susceptible de permettre la réalisation d'un emprunt. Cette idée resta sans suite. Mais l'asssembleé départementale ne se découragea point: en I898, elle invita le Préfet à se mettre en rapports avec ses collègues de la région à l'effet de rechercher les conditions dans lesquelles une entente pourrait intervenir entte les conseils généraux des cépartements intéressés au reboisement du bassin supérieur de la Loire et de l'Allier.

Le conseil général de la Loire était alors présidé par un ardent reboiseur, le Sénateur Audiffred, à ce.moment-là député, dont demandes réitérées provoquèrent une notre de la direction générale des Eaux et Forêts du 8 novembre 1899 invitant à faire procéder dans le plus bref délai aux études nécessaires pour déterminer dans le département de la Loire, " les terrains dénudés et ravinés du bassin supérieur du fleuve de ce nom, dont le reboisement serait utile et la collocation dans un périmètre désirable ».

Peu de temps après, à sa première session, le conseil général, sous l'impulsion de son président qui avait adressé au début de Igoo à tous les présidents de conseils généraux des départements traversés par le fleuve " la Loire ) une circulaire leur suggérant de réclamer la constitution de forêts domaniales dans le bassin supérieur de ce fleuve, fit voter un nouveau vœu tendant à ce que l'Etat consacre à cet effet le dixième au moins du crédit de 3.500 .000 francs inscrit annuellement au budget pour les travaux de gazonnement et de reboisement et étudie, dans le plus bref délai, la création de périmètres de protection.

L'Inspecteur-Adjoint, M. VEssiot, qui avait la responsabilité de la chefferie de Saint-Etienne où il a laissé la trace d'un travail soutenu et efficace, ayant rendu compte des difficultés qu'il aurait à étudier de nouveaux périmètres en raison de son service déjà beaucoup trop chargé, un officier forestier fut spécialement affecté en I90 I au département de la Loire pour cette étude. Ce fut $M$. D'Alverny, qui jusqu'en I9Io marqua de sa puissante personnalité, de sa haute valeur forestière et de sa forte culture son passage dans le département de la Loire.

Mais la volonté de reboisement du conseil général devait se heurter une quarantaine d'ánnées plus tard à la même opposition qui s'était manifestée lors la constitution des périmètres consécutifs à la loi de I860.

L'étude de deux grands périmètres: celui du Gier et celui de la Loire, n'en fut pas moins mise au point, après un travail de plusieurs années qui d'ailleurs ne semble pas avoir donné satisfaction à son auteur M. D'Alverny. Ce dernier a même laissé dans les 'archives de l'Inspection de Saint-Etienne une note datée de septembre I9Io, dans laquelle, après avoir rappelé sa faveur première pour les périmètres projetés, il en critique vivement le principe, leur reprochant de compromettre l'économie pastorale, de n'être 
nullement utiles pour lutter contre la torrentialité et de ne pas être susceptibles à priori de donner un revenu supérieur à celui des pâturages à l'état de landes de bruyères qui forment les terrains à reboiser :

"Les $2 / 3$ de l'emprise (des périmètres), dit-il notamment, sont " propriétés particulières et non pas de grands propriétaires fo" rains, mais au contraire presque totalement de petites propriétés " paysannes. Voilà pourquoi nous croyons criminel de compromet" tre, s'il n'y a pas nécessité grave et démontrée, les moyens d'exis" tence d'une seule famille de montagne. Les terrains périmétrés " en I90I-I903 sont des cultures et des pâturages à vaches, sièges " d'une industrie laitière considérable et qui pourrait l'être en( core. )

En posant ainsi nettement le problème pastoral, D'Alverny avait raison, car la réalisation intégrale des périmètres projetés, qui ne laissaient plus qu'une surface réduite à l'immense zone pastorale des hautes chaumes du Forez, n'aurait pas tardé à entraîner la dépopulation de cette région, indépendamment des mécomptes qu'auraient donnés le reboisement en maints endroits. Ses conclusions, qui tendaient d'abord à la protection de la forêt existante et ensuite à un reboisement limité, étaient rationnelles.

Si comme nous le verrons, la constitution des périmètres n'aboutit que partiellement aux résultats escomptés, l'impulsion du conseil général donna cependant aux reboisements un nouvel essor qui se traduisit par la création en 1904 de deux autres pépinières départementales: celle de La Tuilière, dont la contenance primitive de I,43 ha fut portée à $I, 65$ ha en 1923 , celle de Saint-Nizier-de-Fornas qui, d'abord prévue surtout pour les plantations de la forêt des Hospices de Saint-Bonnet-le-Château avec une surface de o,15 ha, ne tarda pas à être agrandie à sa surface actuelle ( $0,70 \mathrm{ha})$ en vue de la fourniture de plants à d'autres collectivités et à des particuliers.

Le département acceptait ainsi une nouvelle charge financière, qu'il devait assumer par la suite sans défaillance, aidé par des subventions de l'Etat.

Le périmètre du Gier, moins important que celui de la Loire et aussi moins sujet à critiques, fut le premier déclaré d'utilité publique par une loi du 7 août I9Io. Il comprenait $7 \mathrm{I} 7,43$ ha répartis entre les communes de Doizieı, La Terrasse-sur-Dorlay, La Vallaen-Gier et Véranne.

Sa constitution fut suivie aussitôt par la création en I9I I d'une quatrième pépinière départementale de 0,69 ha à Noirétable.

La déclaration d'utilité publique de celui de la Loire ne devait être prononcée que plus tard par une loi du 18 juillet 1916, qui en fixait la surface à 3.604 hectares pour I I communes des Monts du Forez et des Monts de La Madeleine. 
En réalité, ce périmètre de la Loire, pour lequel toutes les notifications avisant les propriétaires intéressés de la déclaration d'utilité publique de leurs parcelles furent préparées à l'inspection de Saint-Etienne, mais ne furent jamais envoyées, ne reçut aucune application. Les reboisements qui furent exécutés dans les communes visées le furent essentiellement à titre de reboisements facultatifs et restèrent limités à de petites surfaces isolées.

Pour le périmètre du Gier, aucune notification de déclaration d'utilité publique ne fut non plus adressée. Mais la constitution de ce périmètre provoqua, sous l'impulsion première de M. ServaIs, Inspecteur à Saint-Etienne, de I9Io à I9I3, d'importants reboisements communaux qui ont été réalisés en majorité de I9I I à I935 et qui se poursuivent même actuellement.

Pourquoi la création du périmètre du Gier entraina-telle la réalisation d'un programme important de reboisements, alors que celle du périmètre de la Loire resta à peu près sans conséquence? Il faut en voir la raison dans le fait que le premier n'apportait aucun trouble à la vie économique locale, alors que la mise en application du second se serait heurtée à des habitudes ancestrales et, comme le faisait remarquer D'ALVERNY lui-même son principal auteur, aurait porté un coup presque mortel à la seule région vraiment pastorale du département de la Loire, celle des pentes du Massif de Pierresur-Haute, c'est-à-dire celle des chalets de montagne encore appelés ( jasseries ) (" burons ) dans le Puy-de-Dôme) où de juin à fin octobre vient estiver le bétail. A l'exception peut-être d'une partie de ceux de Doizieu, les terrains reboisés dans le périmètre du Gier étaient des landes communales de faible valeur, où le bétail ne se rendait que lorsqu'il ne pouvait pas trouver dans les pâturages particulier, une nourriture plus substantielle.

C'est certainement en partie à l'impulsion donnée par la déclaration d'utilité publique du périmètre du Gier qu'il faut attribuer le bel effort de reboisement accompli en dehors de ce périmètre, dans des terrains communaux et d'établissements publics, souvent après création de pépinières volantes (pépinière de Burdignes, pépinière du Priel en forêt communale de Pélussin) par les inspecteurs, chefs de service qui se sont succédé depuis rgro à l'inspection de SaintEtienne: MM. les inspecteurs Servais, Lafond, Roy et Jacques.

Parallèlement aux reboisements des terrains communaux et d'établissements publics, se sont multipliés pendant la même période I9IO-I940, et plus spécialement de I920 à I940, les reboisements particuliers, qui ont alors couvert annuellement une surface non encore atteinte depuis la création du Fonds Forestier National.

\section{$3^{\circ}$ Résultats obtenus}

En dépit de conditions de main-d'œuvre et de crédits souvent difficiles, malgré l'aide des subventions, que le Fonds Forestier $\mathrm{Na}$ - 
tional a rendu plus importantes, l'effort se continue, facilité par la production des pépinières départementales et par celle de petites pépinières volantes.

Le tableau ci-dessous illustre mieux que tout développement la continuité de l'effort de reboisement qui, depuis bientôt une centaine d'années, caractérise le département de la Loire.

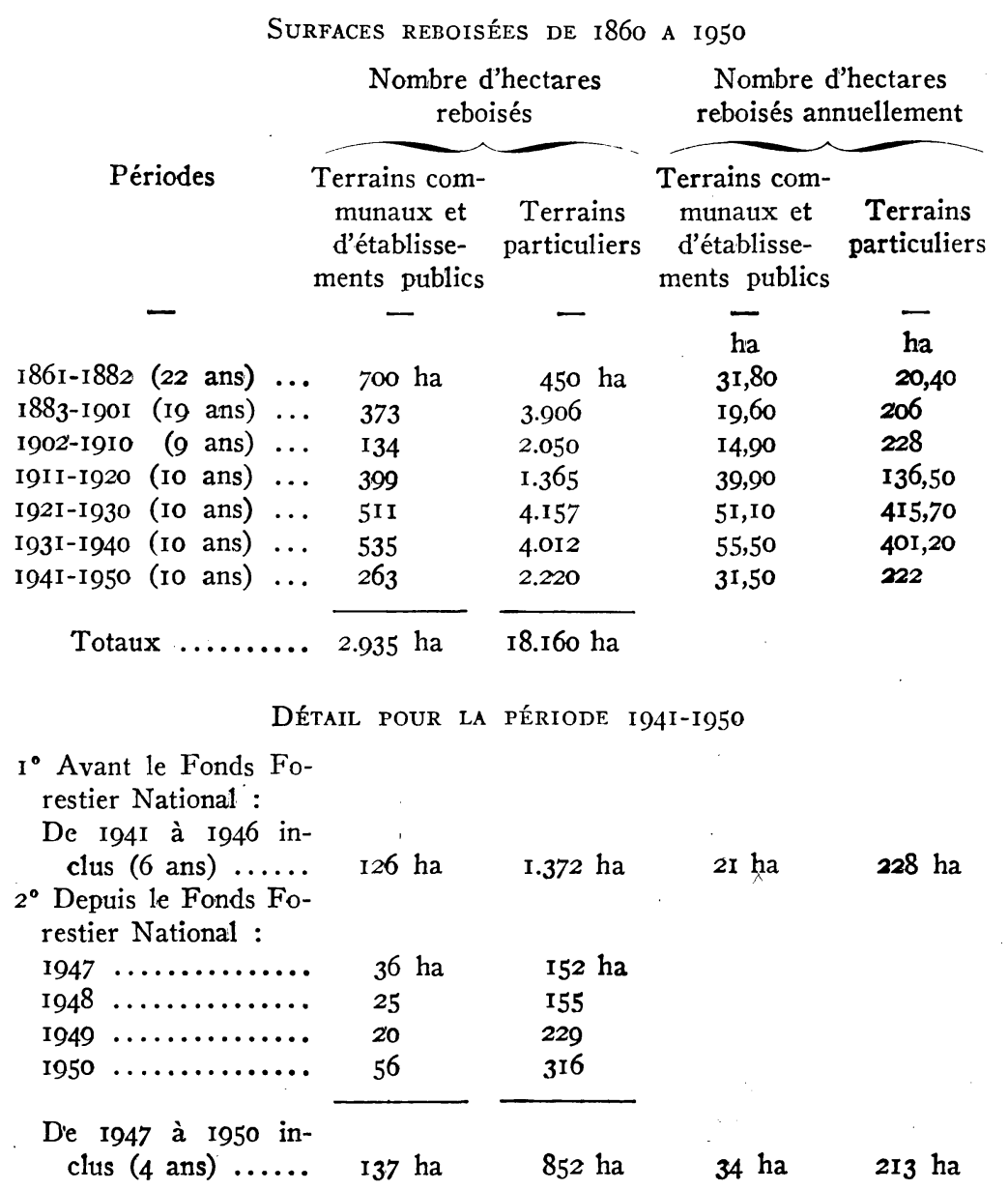

Pour ces reboisements, les pépinières de l'inspection de SaintEtienne ont fourni depuis I863 plus de 83 millions de plants, résineux pour la plupart.

La simple indication, sans commentaires, des chiffres figurant à ce tableau, ne donnerait pas toutefois une image fidèle de la réalité. Il convient en effet de remarquer, en ce qui concerne le tableau des 
reboisements effectués de I86I à I950 inclus, que les surfaces mentionnées comprennent celles qui ont été reboisées à la suite des coupes à blanc-étoc de pins sylvestres, mode normal de traitement de cette essence dans la Loire, ainsi que celles qui, à la suite d'incendies en forêts communales et d'établissements publics, ont été reboisées deux fois, mais que par contre, elles représentent uniquement, pour les terrains particuliers, les surfaces boisées à l'aide de subventions en argent et en nature. Ces deux considérations qui jouent en sens inverse, jointes au fait que, jusqu'à la création du Fonds Forestier National, les plantations particulières subventionnées en nature n'étaient pas l'objet de réceptions, que d'autre part, les plantations particulières subventionnées en argent, n'étaient plus vérifiées par le Service forestier après la réception des travaux, c'està-dire immédiatement après leur exécution, empêchant d'attribuer une valeur absolue aux chiffres indiqués. Ceux-ci donnent néanmoins une idée suffisamment précise de la surface gagnée par la forêt depuis I860, surface que nous évaluons assez approximativement à 20.000 hectares, dont 2.500 au moins pour les forêts communales et d'établissements publics, et qui représente en moyenne près de 225 hectares par an.

\section{II. - Avenir DES REBOisements}

Après cet aperçu historique, il importe maintenant de faire le point de la situation actuelle et de l'avenir des reboisements dans le département de la Loire.

\section{I $^{\circ}$ Possibilités des reboisements}

Une enquête effectuée par le service forestier local en 1920 et I921, à la demande du conseil général (séance du I9 mai I9I9) a fait ressortir une étendue de terrains incultes de 38.949 hectares, dont 4.690 hectares susceptibles de reboisement immédiat sans apporter de troubles de jouissance.

Si l'on se réfère à notre tableau antérieur des surfaces reboisées de I860 à I950, d'où il ressort qu'une surface de I0.389 hectares a été reboisée depuis I92I, l'étendue de 4.690 hectares, qui à cette époque était considérée comme susceptible de reboisement sans apporter de troubles de jouissance, serait entièrement mise en valeur; 5.629 hectares en plus auraient même fait l'objet de plantations et 28.560 hectares resteraient à reboiser, soit environ $6 \%$ de la surface totale du département. Ce dernier chiffre n'est pas incompatible avec les indications de la statistique agricole de I950, qui accuse 59.952 hectares de " landes, pâtis, bruyères ), la différence étant représentée par ce que M. l'Ingénieur Principal Roy appelait “ cette grande étendue de pâturages dégradés eux aussi, envahis par le 
genêt et la bruyère, qui mériteraient mieux le nom de landes que celui de pâturages ).

Il est permis cependant de se demander s'il ne surestime pas la surface susceptible d'être reboisée effectivement. Sans comprendre les landes à bruyère, myrtille et genêt velu qui couvrent les sommets du Massif de Pierre-sur-Haute, il englobe en effet des terrains qui sont superficiels, arides, souvent rocheux et qui se caractérisent par la présence de touffes de Genista purgans (genêt purgatif) ou de Calluna rulgaris (callune). Tels sont de nombreux terrains du bassin du Ban à La Valla-en-Gier, du versant Sud de la commune de St-Paul-en-Jarez, des gorges de la Loire entre le Pertuiset et St-Just-sur-Loire et entre Balhigny et Villerest, des gorges du Vizezy entre Montbrisson et St-Bonnet-le-Courreau, ou encore des gorges du Lignon entre Sail-sous-Couzan et St-Georges-sur-Couzan, terrains clans lesquels il n'est possible d'obtenir que des peuplements élancés, de trop faible valeur pour les dépenses élevées de plantations qu'ils nécessiteraient. Ces terrains ne sont pas certes à abandonner, mais leur reboisement doit être limité aux parties les moins arides.

Les buts du service forestier local doivent, à notre avis, être plus modestes, ce qui ne signifie nullement qu'il n'y ait pas enccre un très gros travail de reboisement à accomplir dans la Loire, plus exactement dans les régions montagneuses du département où, principalement dans le massif du Pilat, dans les parties centrale et septentrionale des Monts du Forez, de même que dans ies Monts de la Madeleine, le reboisement de vastes étendues de médiocres pâtures assurerait un bien meilleur revenu qu'un parcours par le bétail, extensif et souvent limité à une courte période.

Le morcellement de la propriété rend toutefois difficile l'établissement d'un programme d'ensemble de plantations dans les réơions qui paraissent les plus dénudées et sur lesquelles devraient e11 premier lieu porter les efforts. Si, depuis I860, le reboisement s'est pour- . suivi avec une remarquable continuité dans les montagnes de la Loire, il s'est caractérisé essentiellement, tout au moins dans les propriétés particulières, par la dispersion des nouveaux massifs constitués. A l'exception des massifs communaux et d'établissements publics, aujourd'hui soumis au régime forestier, qui ont remplacé d'anciennes landes assez vastes dont ces collectivités ne tiraient qu'un profit limité, ce sont en général de petites parcelles séparées les unes des autres, d'une surface variant de quelques ares à 4 ou 5 hectares qui ont été reboisées. Tout au plus peut-on citer une vingtaine de massifs particuliers d'une certaine étendue qui soient d'origine artificialle.

C'est ce qui explique la présence sur des versants dénudés d'une ou de plusieurs petites taches boisées aux contours souvent très réguliers: un propriétaire se décide, normalement à la suite de difficul- 
tés de main-d'œuvre, à abandonner la culture d'une parcelle de moindre qualité éloignée de son habitation, mais son exemple reste isolé dans la commune ou n'est suivi que par d'autres propriétaires dont les parcelles sont distantes de la sienne. La constitution de périmètres à la suite de la loi de I86o, périmètres dans lesquels d'ailleurs les travaux ont été exécutés, ainsi que nous l'avons vu, sous forme de reboisements facultatifs, n'a pas réussi à provoquer la création de vastes massifs particuliers. Nous avons nous-même essayé d'attirer l'attention des Pouvoirs Publics sur. l'intérêt qu'il y aurait à étudier, en accord avec les services agricoles, deux ou trois communes pour y procéder à une discrimination des sols à vocation agricole et des sols à vocation forestière. Sans avoir encore obtenu un résultat sur ce point, nous pensons néanmoins que des efforts doivent être poursuivis dans ce sens, afin de provoquer des groupements de propriétaires particuliers voisins, dans les parties qui tendent à être abandonnées.

Les communes et les établissements publics ne possédant plus que des étendues très limitées de landes qui ne soient pas nécessaires à la vie pastorale, et ces landes étant elles-mêmes le plus souvent de médiocre qualité, c'est en effet des particuliers qu'il faut attendre le principal effort de reboisement dans l'avenir. Pas l'unique effort cependant, car, dans les régions montagneuses et surtout dans les Monts du Forez et les Monts de la Madeleine, existent d'assez importantes surfaces de terrains sectionaux, provenant de donations d'anciens seigneurs à des hameaux ou à des groupes de hameaux qui sont dans un état de dégradation regrettable, bien que le sol soit souvent d'assez bonne qualité.

Si l'administration forestière est arrivée, par la persuasion, à remplacer la plupart des landes peu productives des communes et des établissements publics, sans nuire d'ailleurs à l'importance du cheptel vif, par des massifs boisés, elle a en général, sauf en ce qui concerne quelques terrains reboisés à la suite de la loi de I860, échoué à l'égard des terrains sectionaux. Et la cause principale en est, à notre avis, le régime même d'administration de ces terrains qui sont gérés par les ayants droit, le plus souvent sans responsables effectifs et sous une tutelle communale plus nominale que réelle. Déjà en I906, M. l'Inspecteur Vessiot écrivait dans un article de la Revue des Eaux et Forêts: "Il serait possible, en améliorant les portions de ces landes qui en sons susceptibles, de maintenir et même d'augmenter le rendement total en foin, tout en reboisant d'énormes surfaces. On ne diminuerait en rien, on accroitrait même les ressources en fourrage nécessaires à l'industrie pastorale et on installerait la forêt sur des étendues considérables où elle rendrait les plus grands services au département $)$.

Là, comme pour les forêts particulières, une certaine insouciance, un certain état d'esprit trop individualiste empêchent d'utiles réalisa- 
tions. On peut le regretter, mais en l'état actuel de la législation, la persuasion et l'exemple de belles réussites peuvent seuls, avec le temps, conduire à des résultats. La prévision d'un programme de mise en valeur par le reboisement des surfaces improductives, ou plus exactement insuffisamment productives, n'est qu'une conception de l'esprit sans l'existence de moyens de coercition applicables et non susceptibles de troubler l'économie locale.

\section{$2^{\circ}$ Réalisations et avenir du F.F.N.}

Les considérations qui précèdent permettent d'expliquer les conséquences dans le département de la Loire de la création du Fonds Forestier National par la loi du 30 septembre 1946 et d'émettre quelques idées sur l'avenir qui lui semble réservé dans ce département. L'absence de vastes étendues de landes communales et d'établissements publics qui ne fassent pas partie intégrante de la vie pastorale, les conditions de gestion des landes sectionnales, le morcellement de la propriété particulière, ne constituent pas à priori, des circonstances favorables à la passation de contrats d'exécution de travaux.

Aussi un très petit nombre de demandes de contrats ont-elles été présentées malgré une propagande réalisée par le recueil des actes administratifs de la préfecture, par quelques causeries et par des conversations avec des maires ou des propriétaires forestiers. Les demandes reçues ont porté d'ailleurs en général sur des terrains de trop faible étendue (4 hectares au maximum) ou d'un reboisement trop aléatoire pour être retenues.

Les prêts n'ont pas davantage jusyu'à présent séduit les propriétaires qui préfèrent très nettement les subventions et surtout les subventions en nature sous forme de plants qu'ils ont l'habitude d'aller prendre eux-mêmes dans les pépinières départementales gérées par le service forestier. Ils évitent ainsi une correspondance avec les pépiniéristes, et les formalités, assez réduites, mais un peu plus importantes, auxquelles donnent lieu les subventions en argent.

Indépendamment du trouble causé par la dernière guerre dans l'exécution des travaux de reboisements, nous attribuons même en partie à la forte diminution de production des pépinières administratives, pendant cette période et pendant les années qui ont immédiatement suivi, la chute constatée de I943 à I949 inclus sur la surface annuelle reboisée.

Les pépinières administratives ne sauraient cependant satisfaire toutes les demandes. Aussi aiguillons-nous vers les subventions en argent les propriétaires qui ont des surfaces importantes à boiser ou qui demandent des plants que nous ne sommes pas en mesure de produire (peupliers), réservant les subventions en nature pour les petites surfaces. En même temps, nous nous efforçons de dévelop- 
per au maximum la production des pépinières autant pour répondre aux desiderata locaux que par mesure d'économie, estimant que la subvention en nature, tout à fait adaptée aux conditions de la propriété rurale du département de la Loire représente la formule de reboisement la moins onéreuse.

Nous espérons arriver avec les surfaces actuelles à une production de 1.200 .000 plants qu'il est difficile de dépasser, les chiffres élevés, accusés pendant les périodes I92 I-I930 et I93 I-I940, ñous paraissant quelque peu surestimés, si nous nous référons aux méthodes alors employées pour le comptage des plants. Désireux d'accroître cette production, qui répond à une incontestable utilité, nous nous proposons de poursuivre par la création d'une ou deux autres pépinières, l'essai très réussi que nous avons fait en I950 en ouvrant à Tarentaize une annexe d'une vingtaine d'ares à la pépinière de Saint-Etienne.

Un effort dans ce sens, est à réaliser notamment dans la région de Roanne, où malgré l'existence de trois pépinières contiguës créées en I935, I937 et I938 par la Société des Amis des Arbres de la Loire, au Domaine des Grands Murcins à Arcon, et aujourd'hui entretenues sur le Fonds Forestier National depuis une décision du 22 mars I949, une autre pépinière se justifierait.

Sur les bases que nous nous sommes fixées: réserver les subventions en argent pour les reboisements les plus importants et les subventions en nature pour les autres, les deux sortes de subventions sont susceptibles de contribuer efficacement au reboisement, sans provoquer de rivalité, mais au contraire en établissant une collaboration étroite entre l'administration et les pépiniéristes. En même temps que s'accroît chaque année le nombre de plants délivrés, s'élève aussi en effet le nombre des subventions en argent qui de

$$
\begin{aligned}
& 86 \text { d'un montant de } 1.869 .300 \text {. francs en } 1948 \\
& \text { et de } 82 \text { - }- \text { de } 2.485 .800-\text { en I949, sont passées } \\
& \text { à I } 47 \quad-\quad-\text { de } 4.936 .000-\text { en I950. }
\end{aligned}
$$

Les propriétaires de la Loire semblent s'intéresser de plus en plus à l'aide apportée par le Fonds Forestier National, sous forme de subventions; des demandes parviennent des régions les plus diverses du département, y compris les régions de la plaine, où le peuplier dans les terrains assez limités toutefois qui lui conviennent, le robinier faux acacia, le chêne, le pin Laricio de Corse, commencent à jouir de la faveur qu'ont toujours rencontrée, dans les parties montagneuses, l'épicéa, le sapin, le pin sylvestre et le douglas. Si la surface reboisée en 1950 (3I6 hectares) n'atteint pas encore celle des périodes I92I-I930 et I93I-I94I, elle est peut-être représentée par des peuplements plus complets, car la subordination du paiement du dernier quart des subventions en argent à une réus- 
site minimum de $70 \%$ incite les propriétaires particuliers à l'exécution de travaux de dégagements et de regarnis qu'ils négligeaient parfois auparavant.

Sans préjuger de l'avenir, nous pensons, bien que pour la première année en 195I nous ayons été saisi, de la part de propriétaires particuliers, de quelques demandes de contrats méritant d'être retenues, que la subvention en nature ou en argent restera l'aide principale du Fonds Forestier National aux propriétaires particuliers reboiseurs du Département de la Loire. Ceux-ci, qui ont en général des étendues de terrains incultes limitées préfèrent en effet aux sujétions des contrats la réalisation d'un programme de plantations subventionnées, à échelonner sur une période de 5 ou 6 ans, lorsque ces étendues atteignent plusieurs hectares. Le contrat ne semble appelé à tenter que les propriétaires qui ne disposent pas des fonds nécessaires pour payer la moitié des travaux dont les dépenses leur incombent ou qui, par suite d'acquisitions de terres abandonnées, se trouveraient à la tête d'un domaine trop vaste pour que le reboisement par subventions soit possible rapidement. Il devrait par contre être le mode normal de reboisement des landes sectionales incultes dont la surface atteint une dizaine d'hectares toutes les fois que l'accord des ayants droit sera obtenu, ceux-ci pouvant plus difficilement assurer la participation en argent aux travaux qu'exige la subvention en argent.

En attendant que des circonstances plus favorables permettent par ce moyen le reboisement progressif de ces landes sectionales, l'augmentation de la surface boisée du département pourra donc être obtenue dans l'immédiat par des subventions en nature et en argent aux particuliers, et en nature aux sections de communes. La formule $\mathrm{du}$ reboisement partiel par subventions semble à préférer à celle du reboisement massif, elle est susceptible d'ailleurs de préparer la voie à des reboisements par contrat.

\section{NiER.}

Un article du même auteur sur la technique des reboisements dans le département de la Loire paraitra dans un prochain numéro.

\section{Nouveau code forestier}

Le Journal Officiel du 30 octobre 1952 publie, pages I0.27I à 10.287 . le décret $\mathrm{n}^{\circ}$ 52-I200 du 29 octobre I952 portant codification des textes législatifs concernant les forêts. 